\title{
Dust-to-gas ratio and star formation history of blue compact dwarf galaxies
}

\author{
H. Hirashita ${ }^{1, \star}$, Y. Y. Tajiri ${ }^{2}$, and H. Kamaya ${ }^{2, \star \star}$ \\ 1 Osservatorio Astrofisico di Arcetri, Largo E. Fermi, 5, 50125 Firenze, Italy \\ 2 Department of Astronomy, Faculty of Science, Kyoto University, Sakyo-ku, Kyoto 606-8502, Japan \\ e-mail: tajiri, kamaya@kusastro.kyoto-u.ac.jp
}

Received 25 July 2001 / Accepted 29 March 2002

\begin{abstract}
This paper investigates the origin of the observed large variety in dust-to-gas ratio, $\mathcal{D}$, among blue compact dwarf galaxies (BCDs). By applying our chemical evolution model, we find that the dust destruction can largely suppress the dust-to-gas ratio when the metallicity of a BCD reaches $12+\log (\mathrm{O} / \mathrm{H}) \sim 8$, i.e., a typical metallicity level of BCDs. We also show that dust-to-gas ratio is largely varied owing to the change of dust destruction efficiency that has two effects: (i) a significant contribution of Type Ia supernovae to total supernova rate; (ii) variation of gas mass contained in a star-forming region. While mass loss from BCDs was previously thought to be the major cause for the variance of $\mathcal{D}$, we suggest that the other two effects are also important. We finally discuss the intermittent star formation history, which naturally explains the large dispersion of dust-to-gas ratio among BCDs.
\end{abstract}

Key words. ISM: dust, extinction - galaxies: dwarf - galaxies: evolution - galaxies: ISM - stars: formation

\section{Introduction}

Dust grains absorb stellar ultraviolet-optical light and emit far-infrared (FIR) light, thereby affecting the spectral energy distributions of galaxies (e.g., Takagi et al. 1999). Since the spectral energy distribution is frequently analysed to infer the star formation history $(\mathrm{SFH})$ that provides us with a key to understand the evolutionary history of galaxies, the research on the origin of the interstellar dust is important to this issue. A key quantity concerning grains is the dust-to-gas mass ratio, $\mathcal{D}$. Oort \& van de Hulst (1946) have observationally shown a strong correlation between the densities of gas and dust. This indicates that dust traces dense environments, which should be rich in heavy elements. Thus, it is worth examining the evolution of $\mathcal{D}$ in the context of the chemical evolution of galaxies.

The condensation of heavy elements is an important process for the formation of grains. One of the environ-

\footnotetext{
Send offprint requests to: H. Hirashita, e-mail: irasita@arcetri.astro.it

* Postdoctoral Fellow of the Japan Society for the Promotion of Science (JSPS) for Research Abroad.

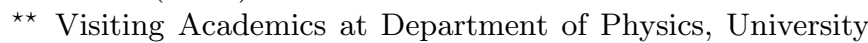
of Oxford, Keble Road, Oxford OX1 3RH, UK (1st March-31st December 2001).
}

ments where condensation takes place is the atmosphere of a cool giant star (Hoyle \& Wickramasinghe 1963). Highdispersion spectroscopic observation of $\mathrm{C}_{2}$ in post AGB stars may indicate the condensation process on $\mathrm{C}_{2}$ as a dust kernel (Crawford \& Barlow 2000; Kameswara Rao \& Lambert 2000). Another environment for condensation is a supernova (SN). Dwek \& Scalo (1980) have shown that $\mathrm{SNe}$ can be the dominant source of dust grains. This is partly because of a rich metal content in SNe. Indeed, a significant amount of dust is observed within hot SN remnants (Dwek et al. 1983; Moseley et al. 1989; Kozasa et al. 1989) and theoretical work by Todini \& Ferrara (2001) has explained some principal features of dust formation in SN 1987A. Hirashita (1999a) has shown that observational dust amounts suggest that $\sim 10 \%$ (the fraction in mass) of the heavy elements ejected from stars condense into dust grains. Since his model prediction reproduces the observed trend between dust-to-gas ratio and metallicity of dwarf galaxies, we adopt this fraction for dust condensation.

The processes of dust destruction should also be considered. As shown in Dwek \& Scalo (1980), dust grains are not only made from heavy elements but also destroyed in SN shocks (see also McKee 1989; Jones et al. 1996) in a cycle of the birth and death of stars. In short, $\mathcal{D}$ of a galaxy reflects its $\mathrm{SFH}$ via the regulation of dust formation and destruction. To investigate what determines 
the value of $\mathcal{D}$, investigation of a star-forming galaxy is the most interesting since $\mathrm{SNe}$ are expected to affect most largely their dust amount.

One of the observational features which give us a key to understand the regulation of $\mathcal{D}$ is the variance of $\mathcal{D}$ itself (e.g., Lisenfeld \& Ferrara 1998, hereafter LF98). In this paper we examine the variance of $\mathcal{D}$ among galaxies. According to LF98, there is a large variance in $\mathcal{D}$ for a sample of blue compact dwarf galaxies (BCDs). By definition, galaxies categorised as BCD more or less show active star-forming activity. Therefore, dust formation and destruction are expected to occur in BCDs. Since grains are composed of heavy elements, application of the theory of galactic chemical evolution is useful and interesting. Then, we particularly consider the variance of $\mathcal{D}$ in BCDs by using the chemical evolution model developed by LF98 and Hirashita (1999b, hereafter H99).

LF98 applied a chemical evolution model to explain $\mathcal{D}$ of dwarf galaxies including BCDs. They have suggested that if the dust-to-gas ratio in outflow (galactic wind) is different from that in the interstellar medium (ISM), the large dispersion of $\mathcal{D}$ of dwarf galaxies can be explained. In this scenario, a significant outflow of gas is indispensable to explain the observed variance of dust-to-gas ratio of BCDs. However, is the variance of $\mathcal{D}$ determined simply by mass outflow? In this paper, after further investigation in the framework of LF98, we answer this question and point out that other factors are also important to explain the value and variance of $\mathcal{D}$.

Indeed, a recent analysis by Tajiri \& Kamaya (2002) has suggested that outflow is not so efficient for BCDs. They estimated the current momentum supply from $\mathrm{SNe}$ by using $\mathrm{H} \alpha$ luminosity, and concluded that the supplied momentum is not sufficient to blow away the $\mathrm{H}$ I envelopes surrounding star-forming regions and that BCDs do not currently suffer significant mass loss. Legrand et al. (2001) also suggested that low density halos around BCDs can be an obstacle for the ISM to escape from the galaxies themselves. Thus, it is worth examining mechanisms other than mass outflow. Since Tajiri \& Kamaya (2002) adopted a sample in Sage et al. (1992), we also use the sample. Moreover, the sample in LF98 is also included because this paper is an extended study of LF98.

This paper is organised as follows. First, in Sect. 2 we explain the model that describes the evolution of dust content in a galaxy. In Sect. 3, we consider dust destruction, which is the most important process in this paper. Then, in Sect. 4, model predictions are presented in comparison with observations. In Sect. 5, we discuss the results and propose a physical mechanism that can explain the observations. Finally, we summarise the contents of this paper.

\section{Model description}

In order to consider the dust formation and destruction, we analyse the dust-to-gas ratio along with the chemical evolution model by H99. The model is based on
Eales \& Edmunds (1996), LF98 and Dwek (1998). In our model, we do not need to model any SFH. This has an advantage in considering the dust-to-gas ratio of BCDs since the SFH of a dwarf galaxy is generally complex (e.g., Grebel 2001) and is difficult to model. We focus especially on dust destruction, because LF98 have not fully considered it. As we see later, our model is a powerful tool to know the metallicity level where dust destruction becomes effective enough to suppress the dust-to-gas ratio.

\subsection{Brief review of the model}

In order to investigate dust content in a galaxy, H99 has established a set of model equations describing dust formation and destruction processes. In H99, a galaxy is treated as one zone to focus on the quantities averaged over the whole galaxy. The galaxy is assumed to be a closed system; that is, mass inflow and outflow are not considered. If the metallicity of the infalling material is zero or much lower than that of the ISM in the galaxy, the relation between dust-to-gas ratio and metallicity, with which we will be concerned in this paper, is not altered by infall (Edmunds 2001; Hirashita 2001). This is because the infall dilutes both metallicity and dust-to-gas ratio at almost the same rate. Our model does not include the effect of outflow, and this is different to LF98, in which outflow is essential to explain the observed variance of the dust-to-gas ratio in BCDs. Since Tajiri \& Kamaya (2002) and Legrand et al. (2001) have suggested that outflow is not efficient for BCDs, it is worth examining a case of no outflow. Indeed, we present another clear possibility to explain the large scatter of $\mathcal{D}$ among BCDs later.

The model equations in H99 (see the paper for details; see also LF98) describe the evolution of total gas mass $\left(M_{\mathrm{g}}\right)$, the total mass of metals (both in gas and dust phases) labeled as $i\left(M_{i} ; i=\mathrm{O}, \mathrm{C}, \mathrm{Fe}\right.$, etc. $)$, and the mass of metal $i$ in a dust phase $\left(M_{\mathrm{d}, i}\right)$. We neglect dust growth in clouds, since Hirashita (1999a) has shown that this process in low-metallicity systems such as dwarf galaxies is much less efficient than the formation of dust around stars. Then, we adopt an instantaneous recycling approximation as in LF98 and H99 according to the formalism in Tinsley (1980): stars less massive than $m_{t}$ (present turn-off mass set to be $1 M_{\odot}$ ) live forever and the others die instantaneously.

\subsection{Solution of the model}

Dust-to-gas ratio and metallicity of galaxies are observationally known to correlate with each other (e.g., Issa et al. 1990). This relation has recently been used as a test for chemical evolution models including dust formation and destruction (LF98; H99; Hirashita 1999a; Edmunds 2001). The model by H99 reduces the following differential equation:

$\mathcal{Y}_{i} \frac{\mathrm{d} \mathcal{D}_{i}}{\mathrm{~d} X_{i}}=f_{\mathrm{in}, i}\left(\mathcal{R} X_{i}+\mathcal{Y}_{i}\right)-\left(\mathcal{R}+\beta_{\mathrm{SN}}\right) \mathcal{D}_{i}$ 
where $\mathcal{D}_{i}$ is the mass fraction of an element $i$ locked up in dust (i.e., $\left.\mathcal{D}_{i} \equiv M_{\mathrm{d}, i} / M_{\mathrm{g}}\right) ; X_{i}$ is the mass fraction of an element $i$ (i.e., $X_{i} \equiv M_{i} / M_{\mathrm{g}}$ ); $f_{\mathrm{in}, i}$ quantifies what fraction of an element $i$ ejected from stars condenses into dust grains; $\beta_{\mathrm{SN}}$ is "dust destruction efficiency", which we explain later; $\mathcal{R}$ is the fraction of stellar mass subsequently returned to the interstellar space, and $\mathcal{Y}_{i}$ is the mass fraction of an element $i$ newly produced and ejected by stars ${ }^{1}$. The definition of $\beta_{\mathrm{SN}}$ is as follows:

$\beta_{\mathrm{SN}}=\frac{M_{\mathrm{g}}}{\tau_{\mathrm{SN}} \psi}$

where $\tau_{\mathrm{SN}}$ is the timescale of dust destruction by SN shocks (Eq. (5)), and $\psi$ is the star formation rate (SFR). Hereafter, $\beta_{\mathrm{SN}}$ is called "dust destruction efficiency", because it is inversely proportional to the timescale of dust destruction $\left(\tau_{\mathrm{SN}}\right)$. This is the most important parameter in this paper.

When all the quantities except $X_{i}$ and $\mathcal{D}_{i}$ are constant in time, the analytical solution obtained by LF98 is applicable. With our notations, it is rewritten as

$\mathcal{D}_{i}\left(X_{i}\right)=\frac{b}{a} X_{i}+\left(1-\mathrm{e}^{-a X_{i}}\right)\left(\frac{c}{a}-\frac{b}{a^{2}}\right)$,

where $a \equiv\left(\mathcal{R}+\beta_{\mathrm{SN}}\right) / \mathcal{Y}_{i}, b \equiv f_{\text {in }, i} \mathcal{R} / \mathcal{Y}_{i}$, and $c \equiv f_{\text {in }, i}$.

Here, we select oxygen as a traced element (i.e., $i=\mathrm{O}$ ) according to LF98, because (i) most of the oxygen is produced by massive stars (Type II SNe and their progenitors), (ii) oxygen is one of the main constituents of dust grains, and (iii) the common tracer for the metal abundance in BCDs is an oxygen emission line. The first item (i) means that an instantaneous recycling approximation may be reasonable for the investigation of oxygen abundances, since the generation of oxygen is a massivestar-weighted phenomenon. In other words, results are insensitive to the value of $m_{t}$. Following H99, we adopt $\left(\mathcal{R}, \mathcal{Y}_{\mathrm{O}}\right)=\left(0.32,7.2 \times 10^{-3}\right)$, which are consistent with the relation between dust-to-gas ratio and metallicity of nearby galaxies including our BCD sample.

\section{Destruction efficiency of dust}

In this paper, we discuss the variance of dust-to-gas ratio of a BCD sample in terms of the variation of $\beta_{\mathrm{SN}}$ defined in Eq. (2). We estimate $\beta_{\mathrm{SN}}$ based on McKee (1989) and LF98, while we also address their differences.

We assume that gas is divided into two components: gas in the star-forming region and that in the $\mathrm{H}$ I envelope. Such an envelope is generally observed around a star-forming region of a BCD (e.g., van Zee et al. 1998). We denote the gas mass fraction in the star-forming region as $X_{\mathrm{SF}}$ and that in the $\mathrm{H}$ I envelope as $X_{\mathrm{HI}}$ (i.e., $\left.X_{\mathrm{SF}}+X_{\mathrm{HI}}=1\right)$. We distinguish the two regions for the comparison with the IRAS sample (Sect. 4.1). IRAS FIR

\footnotetext{
${ }^{1} \mathcal{R}=R$ and $\mathcal{Y}_{i}=y(1-R)$ for the notation in LF98. These two parameters are calculated in the formalism of the instantaneous recycling approximation.
}

bands are sensitive to dust hotter than about $25 \mathrm{~K}$. Such "warm" dust exists in star-forming regions, not in $\mathrm{H}$ I envelopes. Calzetti et al. (1995) have also shown by using the IRAS sample of actively star-forming galaxies that $70 \%$ of the FIR flux comes from such a warm component of dust. Thus, dust mass derived from the IRAS observation of a $\mathrm{BCD}$ is considered to trace the dust in the star-forming region. This suggests that it is useful for us to consider dust contained in star-forming regions as long as we are interested in the comparison of our result with the IRAS observations.

Thus, we estimate $\tau_{\mathrm{SN}}$ and $\beta_{\mathrm{SN}}$ in star-forming regions. Gas mass accelerated to a velocity of $v_{\mathrm{s}}$ by a $\mathrm{SN}, M_{\mathrm{s}}\left(v_{\mathrm{s}}\right)$, is estimated as

$M_{\mathrm{S}}\left(v_{\mathrm{s}}\right)=6800 \frac{E_{51}}{v_{\mathrm{s} 7}^{2}} M_{\odot}$,

where $E_{51}$ is energy released by a SN in units of $10^{51} \mathrm{erg}$ and $v_{\mathrm{s} 7}$ is $v_{\mathrm{s}}$ in units of $10^{7} \mathrm{~cm} \mathrm{~s}^{-1}$ (McKee 1989). In swept ISM, dust is not fully destroyed. Thus, the fraction of destroyed dust, $\epsilon(\sim 0.1$; McKee 1989), should be multiplied. Since we are interested in mass swept by multiple SNe in a star-forming region, SN rate must be considered, which is denoted as $\gamma$. Then, $\tau_{\mathrm{SN}}$ is expressed as

$\tau_{\mathrm{SN}}=\frac{M_{\mathrm{g}} X_{\mathrm{SF}}}{\epsilon \gamma M_{\mathrm{S}}\left(100 \mathrm{~km} \mathrm{~s}^{-1}\right)}$.

Here, we substitute $v_{\mathrm{s}}$ with the threshold velocity for dust destruction (100 $\mathrm{km} \mathrm{s}^{-1}$; McKee 1989). Comparing Eqs. (2) and (5), we obtain

$\beta_{\mathrm{SN}}=\epsilon M_{\mathrm{S}}\left(100 \mathrm{~km} \mathrm{~s}^{-1}\right) \frac{\gamma}{\psi} \frac{1}{X_{\mathrm{SF}}}$.

We should consider both Type Ia and II SNe in estimating $\gamma$. Type II SNe occur "soon" after a star formation, because the progenitors of Type II SNe have lifetimes much shorter than the age of the universe. On the other hand, Type Ia SNe, whose progenitors are low-mass stars, occur about 1 Gyr after a star formation. Thus, $\gamma$ and $\beta_{\mathrm{SN}}$ should be sensitive to the SFH. We express $\gamma$ as

$\gamma=\gamma_{\mathrm{Ia}}+\gamma_{\mathrm{II}}$

where $\gamma_{\text {Ia }}$ and $\gamma_{\text {II }}$ are the rate of Type Ia and Type II SNe, respectively. We note that LF98 estimated $\beta_{\mathrm{SN}}$ to be 5 . But, for example, the fraction between $\gamma_{\mathrm{Ia}}$ and $\gamma_{\mathrm{II}}$ changes according to $\mathrm{SFH}$ of BCDs. In the next section, we examine the relation between dust-to-gas ratio and metallicity for various $\beta_{\mathrm{SN}}$, whose probable range is constrained.

\section{Sample and result}

\subsection{Observational samples}

We compare the model calculation in Eq. (3) with the observed values of dust-to-gas ratio and metallicity of BCDs. We adopt the data in Table 2 of LF98 and Table 1 of Sage et al. (1992). The latter sample has been adopted by Tajiri \& Kamaya (2002). We select BCDs whose 21-cm 
Table 1. Data of blue compact dwarf sample.

\begin{tabular}{lccccccc}
\hline \hline Object & $\begin{array}{c}D \\
(\mathrm{Mpc})\end{array}$ & $\begin{array}{c}S_{60} \\
(\mathrm{Jy})\end{array}$ & $\begin{array}{c}S_{100} \\
(\mathrm{Jy})\end{array}$ & $\begin{array}{c}\log M_{\mathrm{HI}} \\
{\left[M_{\odot}\right]}\end{array}$ & $\begin{array}{c}\log M_{\mathrm{d}}^{I R A S} \\
{\left[M_{\odot}\right]}\end{array}$ & $12+(\mathrm{O} / \mathrm{H})$ & ref. $^{\text {a }}$ \\
\hline II Zw 40 & 9.1 & 6.6 & 5.8 & 8.30 & 4.55 & 8.15 & $\mathrm{~S} 92$ \\
Haro 2 & 20.3 & 4.8 & 5.5 & 8.68 & 5.39 & 8.4 & $\mathrm{~S} 92$ \\
Haro 3 & 13.7 & 5.2 & 6.7 & 8.76 & 5.21 & 8.3 & S92 \\
UM 439 & 12.6 & 0.39 & 1.2 & 8.23 & 4.96 & 7.98 & S92 \\
UM 462 & 11.9 & 0.99 & 1.1 & 8.15 & 4.21 & 7.89 & S92 \\
UM 465 & 13.2 & 0.99 & 1.3 & 7.71 & 4.48 & 8.9 & S92 \\
UM 533 & 10.4 & 0.51 & 0.54 & 7.76 & 3.75 & 8.10 & S92 \\
UM 448 & 6.00 & 4.14 & 4.32 & 7.54 & 4.16 & 8.08 & LF98 \\
IC 3258 & 21.2 & 0.490 & 0.970 & 8.55 & 5.03 & 8.44 & LF98 \\
Mrk 7 & 42.3 & 0.480 & 0.970 & 9.56 & 5.64 & 8.54 & LF98 \\
Mrk 33 & 21.6 & 4.68 & 5.30 & 8.77 & 5.41 & 8.40 & LF98 \\
Mrk 35 & 14.5 & 4.95 & 6.74 & 8.73 & 5.29 & 8.30 & LF98 \\
Mrk 450 & 12.1 & 0.480 & 0.820 & 7.77 & 4.37 & 8.21 & LF98 \\
NGC 4670 & 12.1 & 2.63 & 4.47 & 8.52 & 5.10 & 8.30 & LF98 \\
NGC 4861 & 12.9 & 1.97 & 2.26 & 9.13 & 4.60 & 8.08 & LF98 \\
II Zw 70 & 17.6 & 0.710 & 1.24 & 8.56 & 4.89 & 8.11 & LF98 \\
\hline
\end{tabular}

a S92 - Sage et al. (1992); LF98 - Lisenfeld \& Ferrara (1998).

H I emission and FIR dust emission are both detected. The data are summarised in Table 1.

The observational dust-to-gas ratio in a star-forming region should be

$\mathcal{D}^{\text {obs }} \equiv M_{\mathrm{d}}^{I R A S} /\left(M_{\mathrm{HI}} X_{\mathrm{SF}}\right)$,

where $M_{\mathrm{d}}^{I R A S}$ and $M_{\mathrm{HI}}$ are dust mass determined from the IRAS observation and the total $\mathrm{H}$ I gas determined from the 21-cm observations. $M_{\mathrm{HI}}$ traces $\mathrm{H}$ I gas in both the star-forming region and the envelope of a galaxy. On the other hand, $M_{\mathrm{d}}^{I R A S}$ traces the amount of dust in the star-forming region, because the IRAS is sensitive to hightemperature $(\gtrsim 25 \mathrm{~K})$ dust. In the following two paragraphs, we describe the two quantities further.

LF98 divided the observed H I mass by a factor of 2 to obtain the gas mass in only the star-forming region. In other words, LF98 assumed that $X_{\mathrm{SF}}$ is 0.5 . However, $X_{\mathrm{SF}}$ is hardly constrained observationally. In this paper we assume $X_{\mathrm{SF}}=1$ for the observational sample to obtain the first result. As we discuss in Sect. 5, the variation of $X_{\mathrm{SF}}$ also contributes to the variation of $\beta_{\mathrm{SN}}$. Therefore, $X_{\mathrm{SF}}$ affects our analysis in the following two ways:

1. Observationally, the estimate of dust-to-gas ratio in a star-forming region is affected by the value of $X_{\mathrm{SF}}$.

2 . Theoretically, the value of $X_{\mathrm{SF}}$ affects the value of $\beta_{\mathrm{SN}}$, thus changing the dust-to-gas ratio.

It is difficult to discuss the former quantitatively, because we need a high-resolution spatial map of $\mathrm{H}$ I distribution and a reasonable observational definition of a star-forming region on the map. However, we can discuss the latter because the effect of $X_{\mathrm{SF}}$ on $\beta_{\mathrm{SN}}$ is well determined from Eq. (6).

The observational dust mass, $M_{\mathrm{d}}^{I R A S}$, is derived from the luminosity densities at wavelengths of $60 \mu \mathrm{m}$ and
$100 \mu \mathrm{m}$ observed by IRAS using Eq. (4) of LF98. The $I R A S$ bands are insensitive to the cold $(\$ 20 \mathrm{~K})$ dust that lies out of star-forming regions. Moreover, since the dust in a star-forming region suffers destruction by $\mathrm{SN}$ shocks, we should take into account an efficient destruction in the star-forming region. Therefore, in order to discuss the IRAS sample and the selective dust destruction in starforming regions, we need to define $\beta_{\mathrm{SN}}$ as the dust destruction efficiency (Sect. 3) in star-forming regions as we have done in Eq. (6).

Finally, we hypothesise that the fraction of oxygen contained in dust grains is constant for all the sample BCDs. Following H99, we assume the Galactic composition of the grains:

$\mathcal{D}=2.2 \mathcal{D}_{\mathrm{O}}$

\subsection{Conclusion from our model}

In Fig. 1, we show analytical results calculated according to Eqs. (3) and (9) with $i=\mathrm{O}$ for various values of $\beta_{\mathrm{SN}}$ as solid line $\left(\beta_{\mathrm{SN}}=1\right)$, dotted line $\left(\beta_{\mathrm{SN}}=5\right.$; the case of LF98) and dashed line $\left(\beta_{\mathrm{SN}}=25\right)$, respectively. The black and gray squares indicate the observational samples in Sage et al. (1992) and LF98, respectively. The number ratio of oxygen atoms to the hydrogen atoms is denoted as $(\mathrm{O} / \mathrm{H})$. We convert the mass fraction of oxygen, $X_{\mathrm{O}}$, to $(\mathrm{O} / \mathrm{H})$ for the model prediction, assuming $\log X_{\mathrm{O}}+10.80=12+\log (\mathrm{O} / \mathrm{H})$. In this figure, $f_{\text {in, } \mathrm{O}}=0.1$ is assumed to concentrate on the variation in $\beta_{\mathrm{SN}}$. LF98 have shown a large variety of $f_{\mathrm{in}, \mathrm{O}}$ to explain the large scatter of the relation between $\mathcal{D}$ and $X_{\mathrm{O}}$ in their Fig. 7. Since Hirashita (1999a) has shown that $f_{\text {in, }, ~} \sim 0.1$ reproduces the observed trend between dust-to-gas ratio 


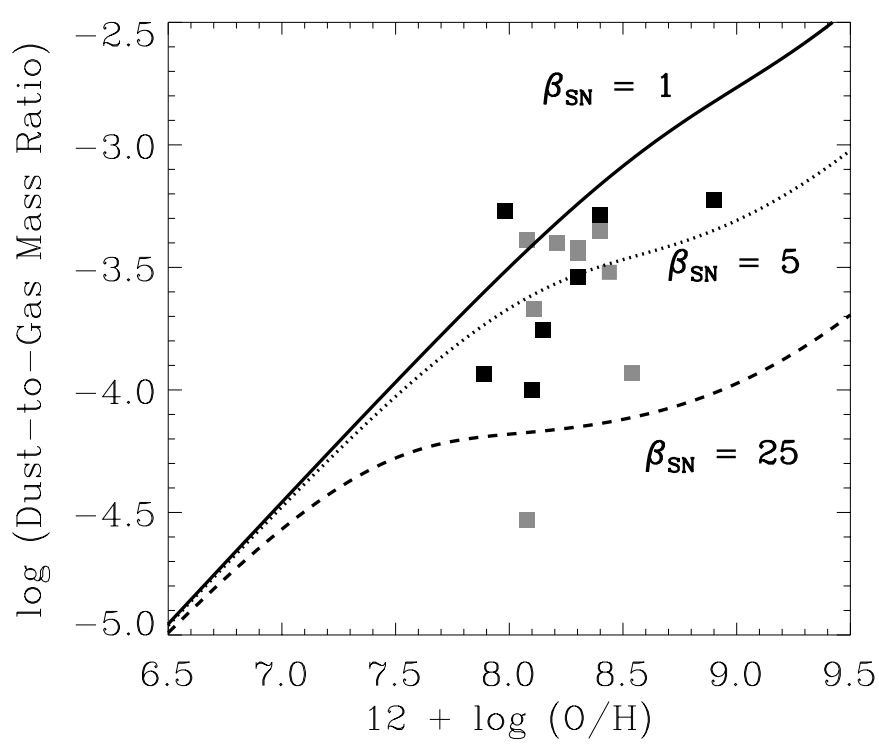

Fig. 1. Relation between dust-to-gas ratio and metallicity. The solid, dotted, and dashed lines show the model results with the dust destruction efficiencies (Eq. (2)) $\beta_{\mathrm{SN}}=1,5$, and 25, respectively. The black and gray squares show the data for the blue compact dwarf galaxies in Sage et al. (1992) and in Lisenfeld \& Ferrara (1998), respectively.

and metallicity, it is worth considering the effect of $\beta_{\mathrm{SN}}$ by setting $f_{\text {in, } \mathrm{O}}=0.1$.

From Fig. 1, we see that the variance in dust-to-gas ratio is reproduced by an order-of-magnitude variation in $\beta_{\mathrm{SN}}(1-25$ here $)$. We note that LF98's value $\left(\beta_{\mathrm{SN}}=5\right)$ is within this range. Even if outflow does not efficiently occur in BCDs, we can explain the variance of the dust-to-gas ratio of BCDs with the various "destruction efficiency" of dust, $\beta_{\mathrm{SN}}$, although we can never reject the importance of outflow in the framework of this paper. The important point is that we have demonstrated that dust destruction by $\mathrm{SNe}$ can play an important role in producing the variance of $\mathcal{D}$. The result also indicates that the difference in dust-to-gas ratio among the three lines becomes clear at an oxygen abundance, $12+\log (\mathrm{O} / \mathrm{H}) \sim 8$. Thus, we conclude

1. that the dust destruction by SNe can vary the dust-togas ratio when the metallicity, $12+\log (\mathrm{O} / \mathrm{H})$, reaches about 8 ( $\sim 10 \%$ of the solar metallicity), and

2 . that the large variation of the dust-to-gas ratio among BCDs is explained by the variation of dust destruction efficiency, $\beta_{\mathrm{SN}}$.

\section{Discussion}

\subsection{Scenario}

We propose a scenario for large variation of $\beta_{\mathrm{SN}}$ for each BCD along with intermittent SFH. The time variability of SFH on a short ( $<1$ Gyr) timescale has been suggested observationally by Searle \& Sargent (1972) and theoretically by Gerola et al. (1980). Nonlinear processes in the ISM may also cause an intermittent star formation (Ikeuchi 1988; Kamaya \& Takeuchi 1997 and references therein). Thus, it is pertinent to consider the intermittent $\mathrm{SFH}$ of BCDs.

As shown in the following, an intermittent star formation history leads to the time variation of $\beta_{\mathrm{SN}}$, because $\gamma / \psi$ depends on time. We consider an intermittent SFH: a starburst whose $S F R$ is $\psi_{\text {burst }}$ and an inter-starburst epoch whose $S F R$ is $\psi_{\text {inter. }}$. We assume that $\psi_{\text {burst }}=100 \psi_{\text {inter }}$, for example. Such a two-orders-of magnitude variation in $S F R$ is proposed theoretically by Gerola et al. (1980) and Kamaya \& Takeuchi (1997). While the starburst is going on, we expect that almost all the SNe are Type II ( $\gamma_{\text {burst }} \sim \gamma_{\mathrm{II}}$, where $\gamma_{\text {burst }}$ is a typical SN rate in the bursting epoch). However, in the inter-burst epoch, Type Ia SNe can be dominated $\left(\gamma_{\text {inter }} \sim \gamma_{\text {Ia }}\right.$, where $\gamma_{\text {inter }}$ is a typical SN rate in the inter-burst). According to the model by Bradamante et al. (1998), a given stellar population releases energy in the form of Type II and Ia SNe with a ratio of 5:1 (Bradamante et al. 1998; see their Fig. 9. This value is essentially determined by the initial mass function (IMF), and they assumed the Salpeter IMF with a stellar mass range from 0.1 to $\left.100 M_{\odot}\right)^{2}$. Since they assumed the same energy between Type Ia and II SNe, this means that the number ratio between Type Ia and II SNe is 5:1. Therefore, we expect that $\gamma_{\text {inter }} \sim \gamma_{\text {burst }} / 5$. The intermittent star formation finally predicts that $\gamma_{\text {inter }} / \psi_{\text {inter }} \sim 20 \gamma_{\text {burst }} / \psi_{\text {burst }}$. This means that an intermittent SFH can cause a 20 -times variation in $\beta_{\mathrm{SN}} \propto \gamma / \psi$ during a single star formation cycle.

Furthermore, Fig. 1 shows that the value of $\beta_{\mathrm{SN}}$ has little effect on the relation between dust-to-gas ratio and metallicity for $12+\log (\mathrm{O} / \mathrm{H})<8$. Therefore, until the metallicity level becomes $12+\log (\mathrm{O} / \mathrm{H}) \sim 8$, the relation between dust-to-gas ratio and metallicity evolves in the same way whatever the value of $\beta_{\mathrm{SN}}$ might be. On the contrary, the relation is largely affected by $\beta_{\mathrm{SN}}$ if $12+$ $\log (\mathrm{O} / \mathrm{H})>8$. Then, we study the response of the relation between dust-to-gas ratio and metallicity to the change of $\beta_{\mathrm{SN}}$ at $12+\log (\mathrm{O} / \mathrm{H}) \sim 8$, as we are interested in the intermittent $\mathrm{SFH}$.

First, we shall estimate a typical metallicity increment during a single star formation epoch of the intermittent SFH. The metallicity increase during an episode of star formation, $\Delta Z$, can be estimated by $\Delta Z \sim y M_{*} / M_{\mathrm{g}}$, where $M_{*}$ is the mass of stars formed in the episode, and $y$ is a chemical yield. If the IMF is similar to that of the Galaxy, $y \sim Z_{\odot}$ (i.e., $\Delta Z \rightarrow Z_{\odot}$ for $M_{*} \rightarrow M_{\mathrm{g}}$ ). We estimate $M_{*}$ by multiplying observed $S F R$ with a duration of an episode of a star formation activity. Assuming that the $S F R$ is $0.1 M_{\odot} \mathrm{yr}^{-1}$ and that the duration is $10^{7} \mathrm{yr}$ (Legrand et al. 2001), we obtain $M_{*} \sim 10^{6} M_{\odot}$. With typical gas mass $M_{\mathrm{g}} \sim 10^{7} M_{\odot}$, we obtain $\Delta Z \sim 0.1 Z_{\odot}$. This corresponds, for example, to the metallicity increase from

\footnotetext{
${ }^{2}$ We assumed the Salpeter IMF with the range from 0.1$120 M_{\odot}$, but the difference in the higher mass cut has little influence on the $\mathrm{SN}$ rate.
} 


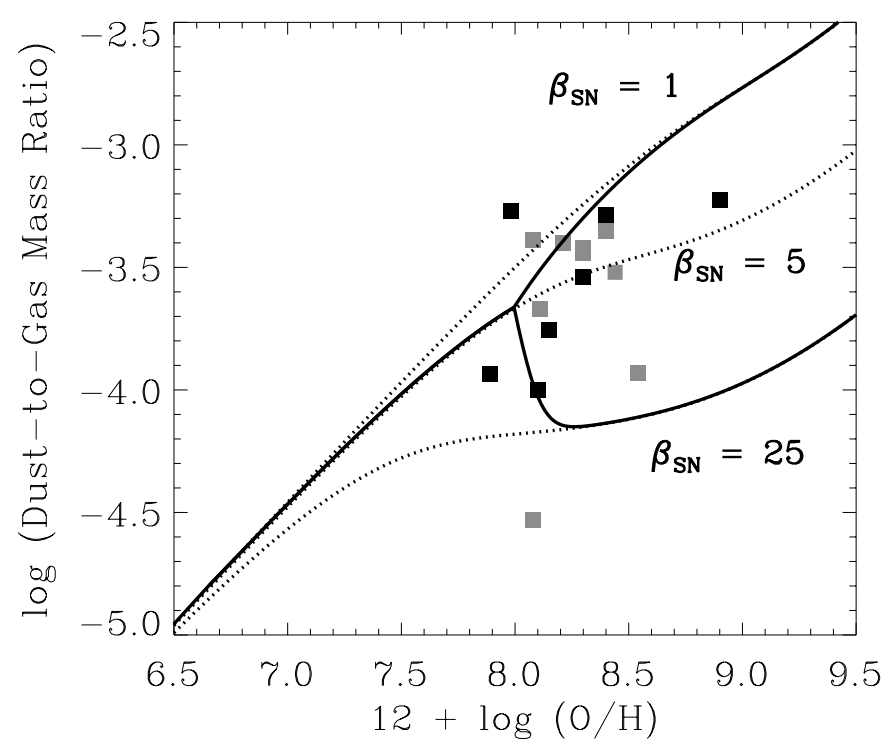

Fig. 2. Relation between dust-to-gas ratio and metallicity. The three dotted lines show the model results same as Fig. 1. The squares are the data points same as Fig. 1. The two solid lines represents the result of the calculations which change $\beta_{\mathrm{SN}}$ (from 5 to 1 and from 5 to 25 for upper and lower lines, respectively).

$12+\log (\mathrm{O} / \mathrm{H})=8.0$ to 8.2 . The model by Bradamante et al. (1998) also indicates that one episode of star formation can result in such a metallicity increment.

As shown above, the effect of intermittence can be examined by changing $\beta_{\mathrm{SN}}$. In order to examine the effect of time variation of $\beta_{\mathrm{SN}}$, thus, we calculate the relation between dust-to-gas ratio and metallicity in the following two cases:

1. $\beta_{\mathrm{SN}}=5$ for $12+\log (\mathrm{O} / \mathrm{H}) \leq 8$ and $\beta_{\mathrm{SN}}=25$ for $12+\log (\mathrm{O} / \mathrm{H})>8$

2. $\beta_{\mathrm{SN}}=5$ for $12+\log (\mathrm{O} / \mathrm{H}) \leq 8$ and $\beta_{\mathrm{SN}}=1$ for $12+\log (\mathrm{O} / \mathrm{H})>8$.

In Fig. 2, we show the result of the two calculations (two solid lines). The lower and upper branches represent the cases 1 and 2, respectively. The three dotted lines show the results as in Fig. 1. We see that when the metallicity $12+\log (\mathrm{O} / \mathrm{H})$ increases from 8.0 to 8.2 , the line rapidly converges to the two dotted lines, which represent the result for constant $\beta_{\mathrm{SN}}$ (1 and 25 , respectively). This rapid convergence supports the idea that the dust-to-gas ratio varies widely in response to the time-evolution of $\beta_{\mathrm{SN}}$ in an episode of star formation at the metallicity level of $12+\log (\mathrm{O} / \mathrm{H}) \sim 8$. Thus, it is possible to determine the variance of the dust-to-gas ratio among BCDs from the time variation of $\beta_{\mathrm{SN}}$.

\subsection{Age difference}

If the age of BCDs varies, the present turn-off mass of stars is changed. As a result, the returned fraction of gas $(\mathcal{R})$, the metal yield $\left(\mathcal{Y}_{i}\right)$, and the dust supply from stars $\left(f_{\text {in }, i}\right)$ are effectively different among BCDs. The effect of varying $\mathcal{R}$ and $\mathcal{Y}_{i}$ on the relation between dust-to-gas ratio and metallicity has been examined by $\mathrm{H} 99^{3}$. However, the resulting relation is less sensitive to the two parameters than to $\beta_{\mathrm{SN}}$. The dependence of $f_{\mathrm{in}, i}$ on the turn-off mass can be important since it largely affects the dust amount in low-metallicity systems (LF98; Hirashita 1999a).

Thus, if the BCD sample proves to have a large age variation, we should reconsider the variation in dust-togas ratio with a time-dependent formulation. Indeed, we cannot reject the possibility that some BCDs are much younger than the cosmic age. A metal-poor BCD SBS 0335-052 may be younger than $5 \times 10^{6}$ yr (Vanzi et al. 2000). Recently, Hirashita et al. (2002) have succeeded in explaining the dust amount of SBS 0335-052 with a timedependent formulation applicable to young galaxies.

\subsection{Future observations}

The time variation of $X_{\mathrm{SF}}$ also leads to the time dependence of $\beta_{\mathrm{SF}}$ (Eq. (6)), although $X_{\mathrm{SF}}$ was assumed as unity (Sect. 4.1). If the various $X_{\mathrm{SF}}$ for the BCD sample is interpreted to reflect the time evolution of $X_{\mathrm{SF}}$ in each BCD, we can suggest that the gas mass in a star-forming region should change temporally because of the mass exchange between the star-forming region and the envelope. Such a mass exchange during episodic star formation activity in BCDs is indeed suggested by e.g., Saitō et al. (2000). The temporal change of $X_{\mathrm{SF}}$ is also possible if the ISM in the star-forming region is consumed for star formation and locked in stellar remnants like white dwarfs, neutron stars, and black holes.

In order to constrain $X_{\mathrm{SF}}$, we need to observe H I emission or FIR emission with angular resolution fine enough. The present typical angular resolution of $1^{\prime}$ corresponds to $2.9 \mathrm{kpc}$ in physical size if a galaxy lies at a typical distance of $10 \mathrm{Mpc}$. Future large space FIR telescopes such as Herschel $^{4}$ (e.g., Pilbratt 2000) or SPICA $A^{5}$ (e.g., Nakagawa et al. 2000) will resolve the star-forming regions of the BCDs. For example, the Japanese future infrared satellite SPICA will have a diameter larger than $3.5 \mathrm{~m}$. If the diffraction limit is achieved, the angular resolution becomes $6^{\prime \prime}$ at the wavelength of $100 \mu \mathrm{m}$. This corresponds to $290 \mathrm{pc}$ at the distance of $10 \mathrm{Mpc}$, and is comparable to or smaller than the half-light radius of a typical BCD (Marlowe et al. 1999).

\section{Summary}

In this paper, we have analysed the relation between dustto-gas ratio and metallicity of BCDs by using our chemical evolution model. We have focused on the dust destruction

\footnotetext{
${ }^{3}$ See Fig. 3 of the paper. Although H99 examined "different IMF", this difference only affects the values of $\mathcal{R}$ and $\mathcal{Y}_{i}$. Therefore, his result can be used to see the effect of varying $\mathcal{R}$ and $\mathcal{Y}_{i}$.

${ }^{4}$ http://astro.estec.esa.nl/First/

${ }^{5}$ http://www.ir.isas.ac.jp/SPICA/index.html
} 
process, because this process was not investigated in LF98. We have shown that the dust destruction significantly affects the dust-to-gas ratio when the metallicity is larger than $12+\log (\mathrm{O} / \mathrm{H}) \sim 8$. The intermittent SFH can explain the large variety of dust-to-gas ratio among BCDs through the "dust destruction efficiency" of $\beta_{\mathrm{SN}}$.

Acknowledgements. We thank the anonymous referee for invaluable comments and suggestions which improved this paper very much. We thank A. Ferrara and K. Yoshikawa for useful discussions on dwarf galaxies and excellent environments for our study. We are also grateful to S. Mineshige and J. Silk for continuous encouragement. H. H. was supported by the Research Fellowship of the Japan Society for the Promotion of Science for Young Scientists. We fully utilised the NASA's Astrophysics Data System Abstract Service (ADS).

\section{References}

Bradamante, F., Matteucci, F., \& D'Ercole, A. 1998, A\&A, 337,338

Calzetti, D., Bohlin, R. C., Kinney, A. L., Storchi-Bergmann, T., \& Heckman, T. M. 1995, ApJ, 443, 136

Crawford, I. A., \& Barlow, M. J. 2000, MNRAS, 311, 370

Dwek, E. 1998, ApJ, 501, 643

Dwek, E., \& Scalo, J. M. 1980, ApJ, 239, 193

Dwek, E., A'Hearn, M. F., Becklin, E. E., et al. 1983, ApJ, 274,168

Eales, S. A., \& Edmunds, M. G. 1996, MNRAS, 280, 1167

Edmunds, M. G. 2001, MNRAS, 328, 223

Gerola, H., Seiden, P. E., \& Schulman, L. S. 1980, ApJ, 242, 517

Grebel, E. K. 2001, Dwarf Galaxies and their Environment, ed. K. S. de Boer, R.-J. Dettmar, \& U. Klein (Shaker Verlag, Bonn), 45

Hirashita, H. 1999a, ApJ, 522, 220

Hirashita, H. 1999b, ApJ, 510, L99 (H99)

Hirashita, H. 2001, Ph.D. Thesis, Department of Astronomy, Kyoto University

Hirashita, H., Hunt, L. K., \& Ferrara, A. 2002, MNRAS, 330, L19

Hoyle, F., \& Wickramasinghe, N. C. 1963, MNRAS, 126, 401
Ikeuchi, S. 1988, Fundam. Cosmic Phys., 12, 255

Issa, M. R., MacLaren, I., \& Wolfendale, A. W. 1990, A\&A, 236, 237

Jones, A. P., Tielens, A. G. G. M., \& Hollenbach, D. J. 1996, ApJ, 469, 740

Kamaya, H., \& Takeuchi, T. T. 1997, PASJ, 49, 271

Kameswara Rao, M., \& Lambert, D. L. 2000, MNRAS, 313, L33

Kozasa, T., Hasegawa, H., \& Nomoto, K. 1989, ApJ, 344, 325

Legrand, F., Tenorio-Tagle, G., Silich, S., Kunth, D., \& Cervino, M. 2001, ApJ, 560, 630

Lisenfeld, U., \& Ferrara, A. 1998, ApJ, 496, 145 (LF98)

Marlowe, A. T., Meurer, G. R., \& Heckman, T. M. 1999, ApJ, 522,183

McKee, C. F. 1989, in Interstellar Dust, ed. L. J. Allamandola, \& A. G. G. M. Tielens (Dordrecht: Kluwer), IAU Symp. 135,431

Moseley, S. H., Dwek, E., Glaccum, W., Greham, J. R., \& Loewenstein, R. F. 1989, Nature, 340, 697

Nakagawa, T., et al. 2000, The Institute of Space and Astronautical Science Report SP No. 14, Mid- and FarInfrared Astronomy and Future Space Missions, ed. T. Matsumoto, \& H. Shibai (ISAS, Sagamihara), 189

Oort, J. H., \& van de Hulst, H. C. 1946, Bull. Astr. Inst. Netherlands, 10, 187

Pilbratt, G. L. 2000, The Institute of Space and Astronautical Science Report SP No. 14, Mid- and Far-Infrared Astronomy and Futhre Space Missions, ed. T. Matsumoto, \& H. Shibai (ISAS, Sagamihara), 211

Sage, L. J., Salzer, J. J., Loose, H.-H., \& Henkel, C. 1992, A\&A, 265, 19

Saitō, M., Kamaya, H., \& Tomita, A. 2000, Mapping the Hidden Universe: The Universe Behind the Milky Way (ASP, San Francisco), ASP Conf. Ser., 218, 305

Searle, L., \& Sargent, W. L. W. 1972, ApJ, 173, 25

Takagi, T., Arimoto, N., \& Vansevičius, V. 1999, ApJ, 523, 107

Tajiri, Y. Y., \& Kamaya, H. 2002, A\&A, in press

Tinsley, B. M. 1980, Fundam. Cosmic Phys., 5, 287

Todini, P., \& Ferrara, A. 2001, MNRAS, 325, 726

Searle, L., \& Sargent, W. L. W. 1972, ApJ, 173, 25

van Zee, L., Skillman, E. D., \& Salzer, J. J. 1998, AJ, 116, 1186

Vanzi, L., Hunt, L. K., Thuan, T. X., \& Izotov, Y. I. 2000, A\&A, 363, 493 